\title{
Electroactive properties of the multilayer films containing Prussian Blue nanoparticles
}

\author{
M. Kolasinska-Sojka*, A. Pajor-Swierzy, and P. Warszynski \\ Jerzy Haber Institute of Catalysis and Surface Chemistry, Polish Academy of Sciences, ul. Niezapominajek 8 30-239 \\ Krakow, Poland
}

\begin{abstract}
In our work we have focused on the incorporation of Prussian Blue nanoparticles (NP) into polyelectrolyte multilayer films (PEM). The main goal of presented studies was to obtain polymer/nanoparticles films with controlled electroactive properties. The amount and ordering of deposited nanoparticles depended on the adsorption conditions of the underlying polymer anchoring layer. In the case of fully charged polyelectrolyte layer, nanoparticles were distributed uniformly. When anchoring layer was not completely charged, strong aggregation of nanoparticles at the surface was observed, causing diminution of current response of studied nanocomposite films. Thus, the selection of the proper formation conditions of nanocomposite films may influence their properties and it may even enhance their response.
\end{abstract}

\section{Introduction}

Layer-by-layer deposition (LbL), which is the procedure of the thin film formation by sequential adsorption of polycation and polyanion layers, is an efficient method for obtaining various materials of welldefined properties [1-4]. The LbL assembly consists of sequential deposition of polyelectrolyte (PE) monolayers or any other charged macromolecular species onto oppositely charged interface [5]. The process is applied in cyclic manner profiting from the surface charge overcompensation which occurs while macromolecular species are adsorbed at the solid-liquid interface [6]. The versatility of multilayer formation process, with respect to variety of support materials and the possibility of incorporation of different functional species into multilayers results in extreme interest in such ultrathin objects $[1-3,7,8]$. Such nanometer thick polymer based materials with inhomogeneities embedded in the polymer matrix possess a number of specific properties, pertaining to their structure, thermodynamics and electronic, spectroscopic, optic, electromagnetic or chemical features [9-11] with potential application in chemistry, (bio)sensing and material science [12]. The properties of the obtained materials depend strongly on the inter particle distances in the matrix [13]. Nano sized particles (NP) can be used as such nonhomogeneities. The clustering of filler particles is in general favored by strong attraction, whereas steric and long-range electrostatic forces may stabilize individual beads. Extensive studies in the field of the nanocomposite materials are ongoing. The main challenge of these studies is the enclosure of monodisperse NP in polymer matrices and tuning the interparticle distances and, respectively, the amount of incorporated NP. Successful solution of the existing problems needs detailed understanding of the interactions between particles, the dynamics of the polymer matrix, and the layer organization. The aim of presented studies was to form polyelectrolyte-Prussian Blue nanoparticles composite films with specific electroactive and electrocatalytic properties and to optimize the deposition conditions in order to control films' structure and properties especially the ability of electrocatalytic detection of hydrogen peroxide by Prussian Blue [14, 15] embedded into polyelectrolyte matrix. Prussian Blue catalyzes the electroreduction and electrooxidation of $\mathrm{H}_{2} \mathrm{O}_{2}$. The reduction process of $\mathrm{H}_{2} \mathrm{O}_{2}$ takes place using the high-spin iron ions, $\mathrm{Fe}^{3+/ 2+}$, whereas the oxidation of $\mathrm{H}_{2} \mathrm{O}_{2}$ is the result of the redox reactions at the low-spin iron ions [16]. Films containing Prussian Blue could be an alternative to complicated and not stable enzyme based sensors used in detection of $\mathrm{H}_{2} \mathrm{O}_{2}$. We fabricated and studied nanocomposites which consisted of negatively charged Prussian Blue (PB) nanoparticles and polycations: polyethyleneimine (PEI) and poly(allylamine hydrochloride) (PAH). The nanoparticle deposition was studied using QCM. These experiments were complemented with AFM measurements to reveal the structure of selected films and electrochemical studies were done using cyclic voltammetry technique. 


\section{Materials and Methods}

Prussian blue nanoparticles were synthesized by the reaction of $\mathrm{FeCl}_{3} \times \quad 6 \mathrm{H}_{2} \mathrm{O}$ and $\mathrm{K}_{4}\left[\mathrm{Fe}(\mathrm{CN})_{6}\right]$ in the presence of $10^{-2} \mathrm{HCl}$ according to the recipe given in [17]. All inorganic salts used to prepare the Prussian blue dispersions, $\mathrm{KCl}$ for voltammetric experiments and hydrogen peroxide were purchased from POCH Gliwice. The polyelectrolytes used in our studies were: polycations: poly(allylamine hydrochloride) (PAH, MW $\sim 70 \mathrm{kDa}$ ), branched poly(ethyleneimine) (PEI, MW 750 $\mathrm{kDa}$ ) and polyanion: polystyrene sulfonate (PSS, MW 70 $\mathrm{kDa}$ ). The QSX 301 gold crystals (Q-Sense, Sweden) were used as the substrates for QCM experiments. Before measurements gold crystals were cleaned with piranha solution for 15 minutes, rinsed with water and then kept in hot water $\left(70^{\circ} \mathrm{C}\right)$ for 30 minutes (Precaution! -this solution is a very strong oxidizing agent and should be handled carefully). The same cleaning procedure was done for silicon wafers which were used as support material for selected films studied with AFM.

Film formation was done by layer by layer deposition. PEI was used as an anchoring layer, PAH - as any other polycation layer and negatively charged Prussian Blue nanoparticles were deposited as anionic layers. Time of deposition was 15 minutes for polycations and 20 minutes for PB. Rinsing between adsorption steps was three times for 2 minutes with water.

The size of the Prussian Blue nanoparticles was determined by dynamic light scattering (DLS) using the Zetasizer Nano ZS from Malvern Instruments Ltd. All measurements were performed at $25^{\circ} \mathrm{C}$. The zeta potential of the Prussian Blue nanoparticles was measured by the laser Doppler velocimetry (LDV) technique using Malvern Zetasizer Nano ZS apparatus.

Quartz Crystal Microbalance experiments were carried out using QCM-D system (Q-Sense AB Västra Frölunda, Sweden). The resonance frequency $f$ of the oscillating sensors depends on the total mass and it changes upon adsorption/desorption of any mass to/from the sensor. The adsorbed mass can be calculated from the Sauerbrey relation [18-20]:

$$
\Delta m=-\frac{c^{*} \Delta f}{n}
$$

Where $\Delta \mathrm{m}$ is the adsorbed mass, $\Delta \mathrm{f}$ is the change in the frequency, $c=17.7 \mathrm{ng} \mathrm{Hz}-1 \mathrm{~cm}-2$ for sensors used in presented studies and $\mathrm{n}$ is a number of overtone used for the experiment oscillation: $n=1,3,5,7 \ldots$

Cyclic Voltammetry (CV) experiments were carried out at stationary conditions using Autolab rotating disk electrode and potentiostat/galvanostat (PGSTAT302N). The electrochemical cell consisted of three electrodes: the gold disk electrode covered with $\mathrm{PE} / \mathrm{PB}$ films acted as the working electrode, the $\mathrm{Ag} / \mathrm{AgCl} / \mathrm{sat} . \mathrm{KCl}$ was the reference electrode and the platinum electrode was used as the counter electrode. Before measurements all solutions were deoxidized by bubbling with laboratory grade Argon (Linde Gas Poland). Gold electrodes were first polished using polishing suspension of aluminum oxide (diameter of $0.05 \mu \mathrm{m}$ ) (from Buehler) and then cleaned with ethyl alcohol (P.O.Ch Gliwice). The polyelectrolyte/Prussian Blue multilayer films were built on the surface of gold electrodes using the LbL technique. In the measurements, the voltage scan rate of $100 \mathrm{mV} / \mathrm{s}$ was used. As the supporting electrolyte $0.1 \mathrm{M}$ potassium chloride was used.

Atomic force microscopy (AFM) images of dried polyelectrolyte multilayers deposited on silicon wafers were obtained with NanoScope IV Multimode atomic force microscope (Veeco, Santa Barbara, CA) working in the tapping mode with silicon cantilevers of nominal spring constant of 40N/m (Veeco).

\section{Results and discussions}

\subsection{Effect of PEI deposition conditions}

As PEI is weak electrolyte, its dissociation degree depends on the $\mathrm{pH}$ value of the solution. Adsorption of PB on fully charged PEI $(\mathrm{pH}=7)$ and partially charged PEI in its natural condition: $\mathrm{pH}=10.5$ was studied. We found, that amount of PB deposited on the layer of PEI deposited from the solution of $\mathrm{pH}=7$ was two times smaller comparing to PEI layer deposited from $\mathrm{pH}=10.5$. (see Fig. 1)

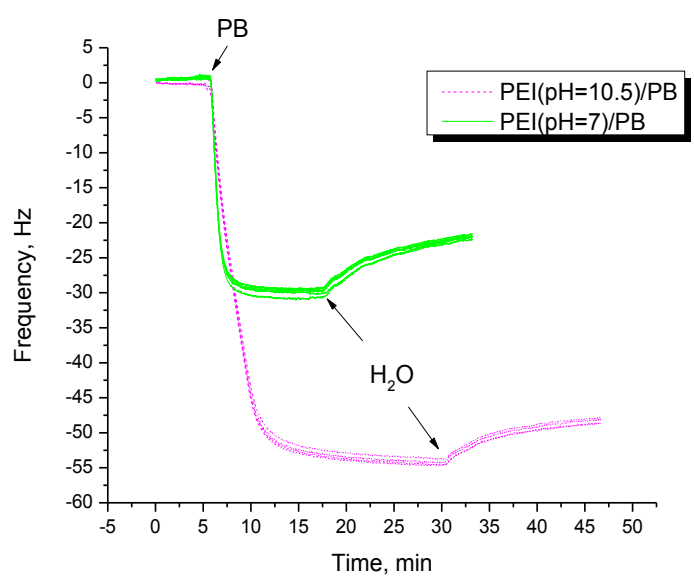

Fig. 1. Frequency shift upon Prussian Blue adsorption on QCM crystal modified with monolayer of PEI deposited from solution of $\mathrm{pH}=7$ (solid line) or $\mathrm{pH}=10,5$ (dashed one); all measured overtones included

Both types of nanocomposites were then used for voltammetric measurements, which are depicted in Fig.2. Voltammetry confirmed the significant influence of the anchoring layer on the electrochemical activity of the studied films. Surprisingly, in the case of films, where PB nanoparticles were deposited on a layer of PEI adsorbed on a gold electrode from a solution of $\mathrm{pH}=10.5$, currents, both oxidation and reduction, are smaller by factor of 2 than for the case of films with PEI deposited from a solution of $\mathrm{pH}=7$. Voltammogram of 3-layer PEM (PEI/PSS/PAH) was also collected as a reference system. One can see no electrochemical response from film without PB nanoparticles. 


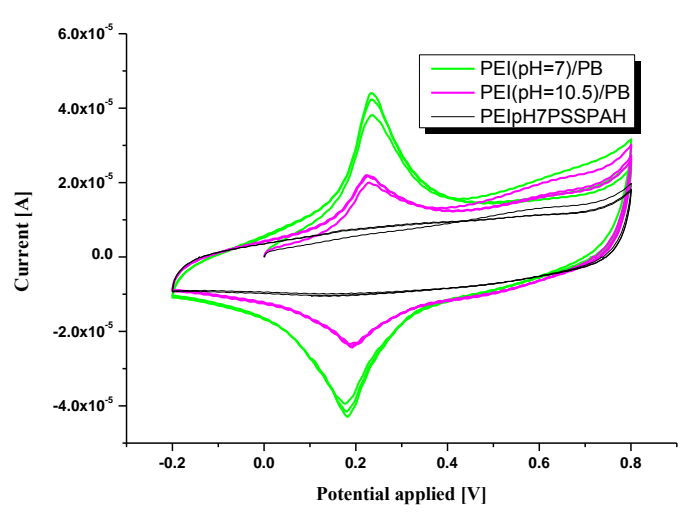

Fig. 2. Voltamogramms of gold electrodes modified with bilayer PEI/PB; PEI deposited from solution of $\mathrm{pH}=7$ (green curve), $\mathrm{pH}=10.5$ (pink curve); voltammogram of 3-layer PEM as reference system (black curve) measured against $\mathrm{Ag} / \mathrm{AgCl} / \mathrm{sat} . \mathrm{KCl}$.

To explain the inconsistency between QCM and CV results, studied nanocomposites were imaged with AFM (see Fig.3). First of all the irreversible adsorption of PB nanoparticles on the layer of PEI was proved with AFM pictures. Moreover, analysis of the AFM images revealed, that nanoparticles deposited on the PEI adsorbed from the solution of $\mathrm{pH}=7$ kept their size (c.a. $10 \mathrm{~nm})$ comparing to one measured directly in the suspension of nanoparticles. In opposition, an average diameter of nanoparticles deposited on PEI $\mathrm{pH}=10.5$ was more than 3 times bigger, although deposition took place from the suspension of PB of $10 \mathrm{~nm}$ in diameter. In this second case, aggregation of the nanoparticles on the surface occurred. Probably this surface aggregation affected the electroactive properties of PB nanoparticles. These aggregates decreased active surface area of Prussian Blue, and consequently - reduced the electrochemical activity of about $50 \%$.

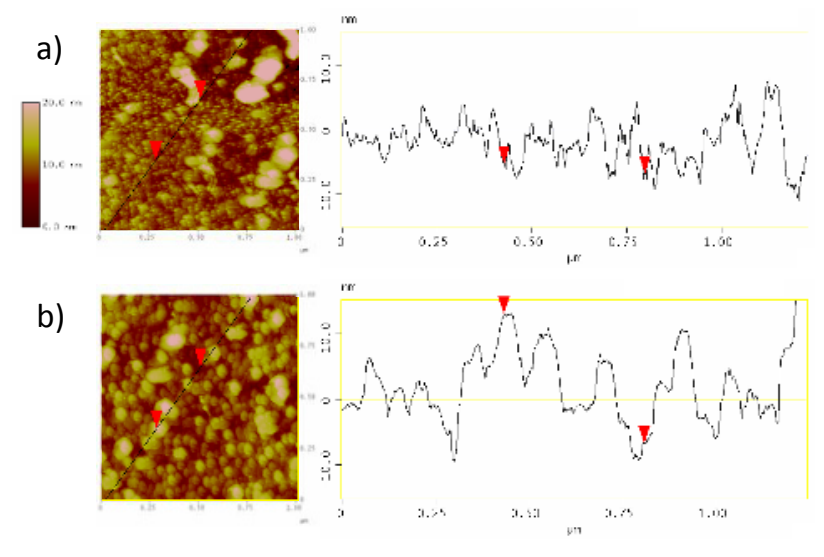

Fig. 3. AFM images and Z-profiles of bilayer nanocomposites: $\mathrm{PEI} / \mathrm{PB}$, PEI deposited from solution of (a) $\mathrm{pH}=7$ or (b) $\mathrm{pH}=10,5$.

\subsection{Effect of ionic strength of polycation solution}

As an ionic strength used for formation of PEM is a key factor determining the structure of obtained multilayers [21], we decided to check how it would affect the properties of the studied electroactive materials. The effect of ionic strength used during deposition of polycation layer on the structure and current characteristics of the hybrid films was studied in the range of 0.015 to $1.5 \mathrm{M} \mathrm{NaCl}$. In Fig. 4 masses calculated from QCM frequency shifts for all studied cases are presented. One can observe, that amount adsorbed depends on the ionic strength used.

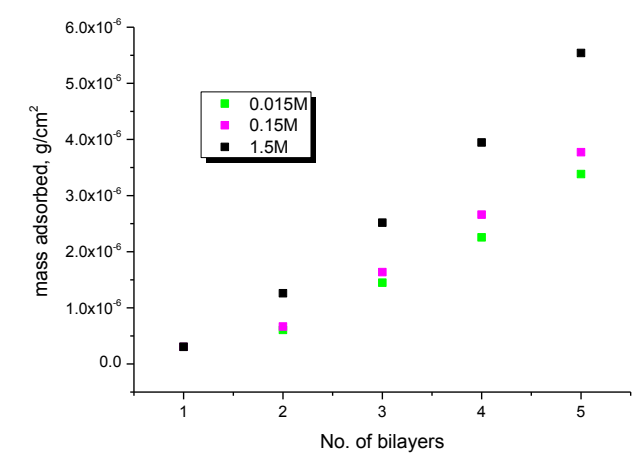

Fig. 4. Mass adsorbed upon the deposition of 5-bilayer PB/PAH films on QCM crystal modified with monolayer of PEI deposited from solution of $\mathrm{pH}=7$; $\mathrm{PAH}$ adsorbed at three different ionic strengths: $0.015 \mathrm{M} \mathrm{NaCl}$ (green curve), $0.15 \mathrm{M}$ $\mathrm{NaCl}$ (pink one), $1.5 \mathrm{M} \mathrm{NaCl}$ (black one).

The higher the ionic strength during the film formation is, the bigger mass of $\mathrm{PB}$ is adsorbed. Ionic strength is crucial for polyion deposition [1]. The higher ionic strength is, the stronger screening of charges along PE chains is observed causing more coiled conformation of $\mathrm{PE}$ chains. It results in bigger thickness of final polyelectrolyte film and bigger mass of polyelectrolytes is adsorbed. Thus, there are more available places for PB nanoparticles to adsorb which explains bigger amount of PB deposited in higher ionic strength. Moreover, in all studied cases one can observe a linear increase of mass with increasing number of layers.

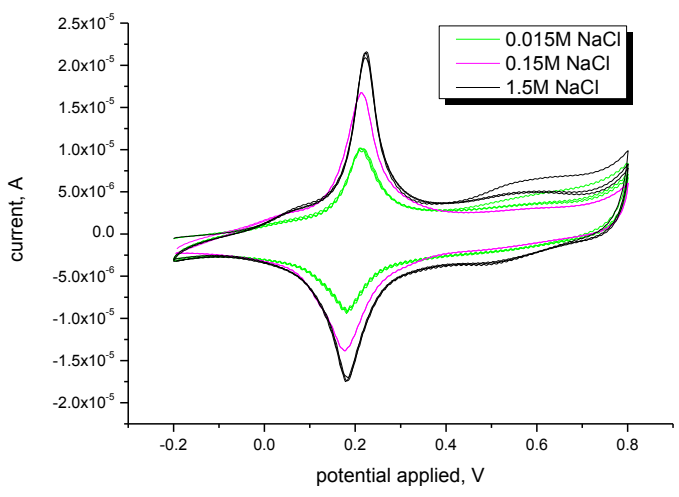

Fig. 5. Voltammograms of gold electrodes modified with four bilayer (polycation/PB) films, where first polycation layer is PEI, each subsequent is PAH deposited from solution of ionic strength: $0.015 \mathrm{M} \mathrm{NaCl}$ (green curve), $0.15 \mathrm{M} \mathrm{NaCl}$ (pink curve) and $1.5 \mathrm{M} \mathrm{NaCl}$ (black curve) measured against $\mathrm{Ag} / \mathrm{AgCl} / \mathrm{sat} . \mathrm{KCl}$. 
CV measurements of all studied series depending on ionic strength of polycation solution were performed. In Fig. 5 an example voltammograms of four bilayer films (polycation/PB), where first polycation layer is PEI, each subsequent is PAH deposited from three studied ionic strengths are depicted. One can observe, that the higher the ionic strength used during the film formation is, the bigger currents are generated by the target structures. Similar experiments were done for series of films containing from 1 to 7 bilayers deposited as described above. For all studied ionic strengths one can see linear increase in oxidation and reduction currents with increasing number of layers (see Fig. 6), which is in agreement with QCM results: higher amount of PB adsorbed generates higher currents.

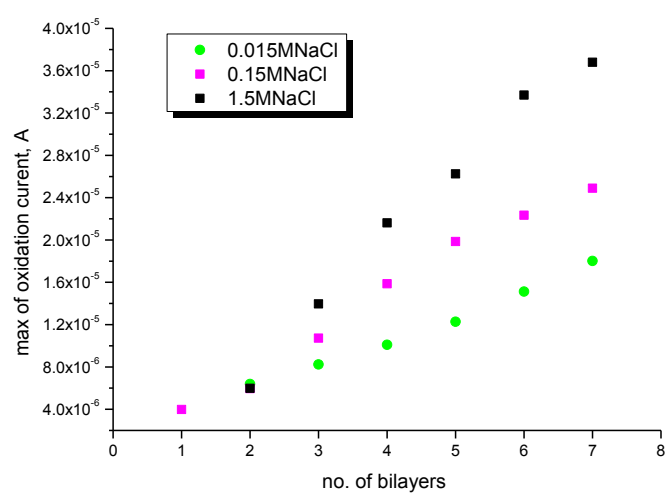

Fig. 6. Maximum of anodic current for series polycation/PB, where first polycation layer is $\mathrm{PEI}$, each subsequent is $\mathrm{PAH}$ deposited from ionic strengths: $0.015 \mathrm{M} \mathrm{NaCl}$ (green plot), $0.15 \mathrm{M} \mathrm{NaCl}$ (pink plot) and $1.5 \mathrm{M} \mathrm{NaCl}$ (black plot) measured against $\mathrm{Ag} / \mathrm{AgCl} / \mathrm{sat} . \mathrm{KCl}$.

\section{Conclusions}

In our work we have focused on the incorporation of Prussian Blue nanoparticles (NP) into polyelectrolyte multilayer films (PEM). We studied formation and electroactive properties of multilayer films of polycations and Prussian Blue nanoparticles. Nanocomposites were formed using layer-by-layer sequential adsorption. Multilayer deposition was proved with QCM experiments of in situ sequential deposition of polycation and PB on quartz crystal. Effect of PEI ancoring layer on film's properties was studied. The amount and ordering of deposited nanoparticles depended on the adsorption conditions of the underlying polymer anchoring layer. In the case of fully charged PEI layer, Prussian blue nanoparticles were distributed uniformly. Oppositely, when anchoring layer was not completely charged, strong aggregation of nanoparticles at the surface was observed causing diminution of current response of studied nanocomposite films, although the mass of prussian blue deposited on not fully charged PEI ( $\mathrm{pH}=10.5)$ was twice as big comparing to PB adsorbed on fully charged PEI layer. Thus, the selection of the proper formation conditions of nanocomposite films may influence their properties and it may even enhance their response.
Effect of ionic strength of polycation solution during deposition was also studied. Three ionic strengths were considered: $0.015 \mathrm{M}, 0.15 \mathrm{M}, 1.5 \mathrm{M} \mathrm{NaCl}$. In all studied cases we observed that linear increase of mass with each subsequent deposited bilayer (as a bilayer we assumed layer of polycation+layer of prussian blue nanoparticles). Comparing nanocomposites of the same number of layers there was a difference in adsorbed mass in dependence on ionic strength used. The higher ionic strength during the deposition of polycation was, the bigger mass was adsorbed. Similar dependency was observed for current responce. First of all, for all studied ionic strengths one can see linear increase in oxidation and reduction currents with increasing number of bilayers (i.e. Prussian blue layers). Moreover, the higher ionic strength during polycation deposition was used, the bigger currents were generated with the most meaningful difference seen for 7 bilayer films.

\section{Acknowledgements}

The work was supported by the HOMING PLUS PROGRAMME co-financed by the Foundation for Polish Science, as part of INNOVATIVE ECONOMY OPERATIONAL PROGRAMME 2007-2013. Authors are grateful to Dr. habil. Szczepan Zapotoczny from the Faculty of Chemistry, Jagiellonian University for AFM images.

\section{References}

1. G. Decher, J.B. Schlenoff, Multilayer Thin Films, Wiley-VCH, Weinheim (2003)

2. P. Bertrand, A. Jonas, A. Laschewsky, R. Legras, Macromol. Rapid Commun., 21, 319 (2000)

3. M. Schönhoff, J. Phys.: Condens. Matter, 15, R1781 (2003)

4. P. T. Hammond., Curr. Opin. Colloid Interface Sci., 4, 430 (2000)

5. G. Decher, Science, 277, 1232 (1997)

6. F. Caruso, H. Möhwald, J. Am. Chem. Soc. 121 6039 (1999)

7. X. Shi, M. Shen, H. Möhwald, Prog. Polym. Sci., 29, 987 (2004)

8. D. Grigoriev, D. Gorin, G. B. Sukhorukov, A. Yashchenok, E. Maltseva, H. Möhwald, Langmuir, 23, 12388 (2007)

9. M. D. Musick, D. J Pena, S. L Botsko, Langmuir, 15, 844 (1999)

10. S. Hrapovic, Y. Liu, G. Enright, Langmuir, 19, 3958 (2003)

11. L. Rivas, S. Sanchez-Cortes, J. V. Garcia-Ramos, G. Morcillo, Langmuir, 17, 574 (2001)

12. S. Rifai, C. A. Breen, D. J. Solis, T. M. Swanger, Chem. Mater. 18, 21 (2006)

13. J. Oberdisse, Soft Matter 2, 29 (2006) 
14. A.A. Karyakin, O.V. Gitelmacher, E.E. Karyakina, Anal. Lett., 27, 2861 (1994)

15. A.A. Karyakin, E.E. Karyakina, L. Gorton, Talanta, 43, 1597 (1996)

16. X. Liu, J.L. Zweier, Biol. Med., 31, 894 (2001)

17. K. Itaya, T. Ataka, S. Toshima, J. Am. Chem. Soc. 104, 4767 (1982)

18. G. Z. Sauerbrey, Phys., 155, 206, (1959)

19. Höök, F., Rodahl, M., Brzezinski, P., Kasemo, B., Langmuir, 14, 729 (1998)

20. M. Rodahl, F. Höök, A. Krozer, P. Brzezinski, B. Kasemo, Rev. Sci. Instrum., 66, 3924 (1995)

21. M. Kolasińska, PhD Thesis, Krakow, 2006 